\title{
New WHO Guidelines on Symptom Based Childhood Contact Screening For Tuberculosis: Relevance to National Tuberculosis Program of India
}

\author{
Divya Nair and Palanivel $\mathrm{C}^{*}$
}

Department of Preventive and Social Medicine, Jawaharlal Institute of Postgraduate Medical Education and Research (JIPMER), Puducherry, India

"Corresponding author: Dr. Palanivel C, MD, Department of Preventive and Social Medicine, Jawaharlal Institute of Postgraduate Medical Education \& Research (JIPMER), Dhanvantri Nagar, Puducherry 605006, India, Tel: 9787825827; E-mail: palaniccm@gmail.com

Received date: May 23, 2014, Accepted date: Jun 30, 2014, Published date: Jul 03, 2014

Copyright: ( 2014 Palanivel C, et al. This is an open-access article distributed under the terms of the Creative Commons Attribution License, which permits unrestricted use, distribution, and reproduction in any medium, provided the original author and source are credited.

\section{Rapid Communication}

Globally there is a growing emphasis on efforts to identify and treat the "missed millions" of Tuberculosis (TB) cases. In India, $5 \%$ of the new TB cases are in the pediatric age group [1] and studies have reported that $3.2-16.2 \%$ of child contacts have TB disease [2]. In view of the susceptibility of younger children to developing TB of increased severity on contact with an active case of tuberculosis, screening of childhood contacts is recommended by the WHO and is also part of most National Tuberculosis Program (NTP) guidelines. Contact screening helps in identifying contacts of all ages with undiagnosed TB disease among the contacts of an index case, and in providing preventive therapy for childhood contacts without TB disease. However, diagnosis of $\mathrm{TB}$ in children is often missed due to the complexities in screening and diagnosis under field conditions.

According to existing NTP guidelines in India, the diagnosis of TB among child contacts is dependent on Tuberculin Sensitivity Test (TST) and chest radiography apart from strong clinical suspicion. WHO also recommended TST and chest radiography for screening childhood contacts for tuberculosis. The implementation of contact screening has been sub-optimal as reported by various studies [2-4], in resource constrained settings like India. Scarcity of diagnostic modalities at primary health care levels, lack of awareness among health workers regarding contact screening and low importance given to the reporting of screening related data in the routine reporting formats [2-4] were the common reasons reported for poor implementation of contact screening among childhood contacts. The recently released WHO guidelines on pediatric TB management recommends that symptom based approach alone is sufficient for screening child contacts [5] thus removing the dependence on TST for contact screening as per previous WHO recommendation. The common symptoms listed are cough, fever, anorexia, weight loss, failure to thrive, fatigue and reduced playfulness $[5,6]$. Though this recommendation is based on a study done in South Africa [6], this approach is simple to implement without dependence on TST and chest radiography, larger number of contacts can be screened and the delay in starting the asymptomatic contacts on Isoniazid Preventive Therapy (IPT) can be reduced.

As per new WHO guidelines, only those children found to be symptomatic based on symptom based screening approach will be evaluated using TST and radiography. The prevalence of the symptoms in general in Indian setting among children may be higher especially due to higher levels of under nutrition prevalent in Indian setting [7]. Symptom checklist used in South Africa for contact screening has to be modified for Indian setting as the two settings are different in respect to HIV prevalence and malnutrition levels. Hence, operational research on symptom based screening will be helpful to establish the feasibility and usefulness of these recommendations in Indian settings. Though WHO has made this recommendation as conditional one with very low quality of evidence, it is important to test this algorithm for childhood contact screening in India to diagnose and prevent more cases of tuberculosis among children.

The WHO guidelines also recommend specific documentation of contact screening data to strengthen the process. Rekha et al. (2013) reported that in programme settings in Tamil Nadu, a state in India, provision of a separate IPT card and register, significantly improved the implementation of screening and provision of IPT for pediatric TB contacts [3]. Along with emphasis on drug resistance TB and TB/HIV, the much neglected contact screening among childhood contacts deserves attention from the national programmes to achieve global TB control.

\section{References}

1. TB India (2014) Annual status report of the Revised National TB Control Programme. Govt of India.

2. Banu Rekha VV, Jagarajamma K, Wares F, Chandrasekaran V, Swaminathan S (2009) Contact screening and chemoprophylaxis in India's Revised Tuberculosis Control Programme: a situational analysis. Int J Tuberc Lung Dis 13: 1507-1512.

3. Rekha B, Jagarajamma K, Chandrasekaran V, Wares F, Sivanandham R, et al. (2013) Improving screening and chemoprophylaxis among child contacts in India's RNTCP: a pilot study. Int J Tuberc Lung Dis 17: 163-168.

4. Pothukuchi M, Nagaraja SB, Kelamane S, Satyanarayana S, Shashidhar, et al. (2011) Tuberculosis contact screening and isoniazid preventive therapy in a South Indian district: operational issues for programmatic consideration. PLoS One 6: e22500.

5. Guidance for National Tuberculosis Programmes on the Management of Tuberculosis in Children (2nd edn), World Health Organisation 2014.

6. Kruk A1, Gie RP, Schaaf HS, Marais BJ (2008) Symptom-based screening of child tuberculosis contacts: improved feasibility in resource-limited settings. Pediatrics 121: e1646-1652.

7. National Family Health Survey (NFHS-3), 2005-06: India: Volume I. International Institute for Population Sciences (IIPS) and Macro International. 2007. 